\title{
Mediastinal cryptococcoma
}

\author{
P SINHA, ${ }^{1} \mathrm{~K}$ G NAIK, ${ }^{2}$ AND G P BHAGWAT ${ }^{2}$ \\ From the Department of Surgery ${ }^{1}$ and Department of Pathology and Microbiology, ${ }^{2}$ \\ University Teaching Hospital, School of Medicine, University of Zambia, Lusaka, Zambia
}

\begin{abstract}
Sinha, P, Naik, K G, and Bhagwat, G P (1978). Thorax, 33, 657-659. Mediastinal cryptococcoma. This communication deals with an unusual presentation of cryptococcoma as a mediastinal mass infiltrating the airways and great vessels.
\end{abstract}

\section{Case report}

A 25-year-old Zambian man was admitted to the University Teaching Hospital (UTH), Lusaka, with a history of productive cough and chest pain for six months and a homogenous mass in the right upper mediastinum in the chest radiograph (fig 1). The chest pain was pricking in nature, continuous, and localised to the right upper chest. The patient had no dyspnoea but admitted to haemoptysis a month before admission. He had neither fever nor night sweats. He also gave a history of a similar episode of chest pain and weight loss five years earlier when he was treated with antituberculous chemotherapy and his symptoms subsided. Before his referral to UTH he had a course of treatment

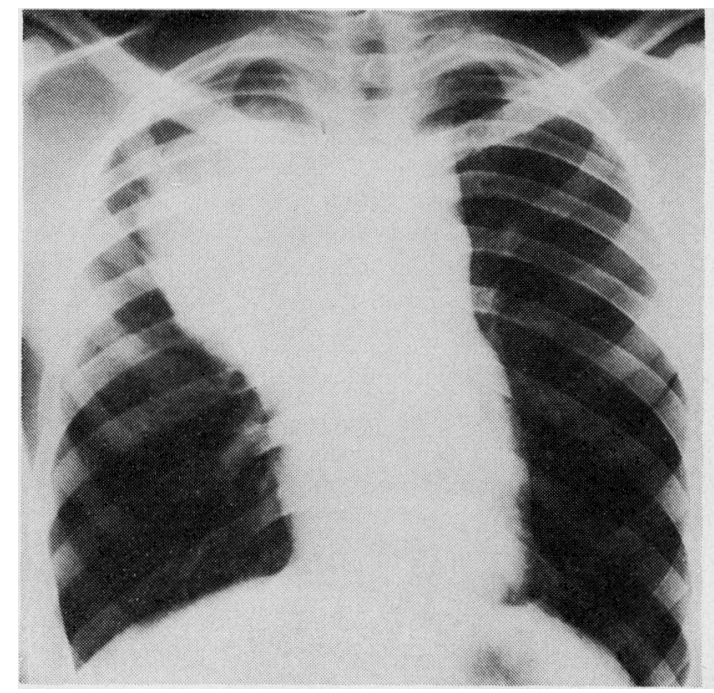

Fig 1 Chest radiograph showing mediastinal mass. with streptomycin and thiazina without improvement; an enlargement of the mass on repeat radiograph of the chest was noted. An attempted bronchoscopy was abandoned because the patient developed severe bronchospasm.

The important physical findings on admission were evidence of recent weight loss and a visible swelling of the anterior aspect of the right upper chest wall. The area was dull on percussion, and bronchial breath sounds were heard over it. There was no evidence of anaemia or peripheral lymphadenopathy.

The haemoglobin was $11.0 \mathrm{~g} / \mathrm{dl}$, total white cell count $11300 / \mathrm{mm}^{3}\left(11.3 \times 10^{9} / 1\right)$, and the erythrocyte sedimentation rate $55 \mathrm{~mm}$ in first hour (Westergren method). The results of sputum examination for acid-fast bacilli, fungi, and abnormal cells were negative on three occasions. Anterior, lateral, and oblique chest radiographs showed a large, well-circumscribed homogenous mass in the anterior mediastinum with evidence of pressure on the trachea by the mass. In view of the weight loss, an enlarging mass, and the absence of tubercle bacilli in the sputum, diagnostic thoracotomy was planned. On induction of anaesthesia it was found impossible to ventilate the patient through an endotracheal tube. When a bronchoscope was passed with difficulty into the left main bronchus, the trachea was found to be completely flat. Ventilation of the left lung was possible, through the bronchoscope, but the patient went into cardiac arrest, and all attempts at resuscitation, including open cardiac massage, failed.

\section{Necropsy findings}

The significant post-mortem findings were bleeding from the nostrils and bulging of the right anterior aspect of the chest. On internal examination a 
mass measuring $13 \times 8.5 \mathrm{~cm}$ was found in the superior mediastinum. It was unencapsulated, firm, greyish, and mucoid in places. The mass compressed the trachea and the right and left main bronchi. It infiltrated the trachea, right and left main bronchus, the hilum of both lungs, the right upper lobe, ascending aorta, pericardium, and outer part of the myocardium. The mucosa of the respiratory tract and the intima of the aorta were free from invasion. The lungs were congested and oedematous.

All other systems of the body including the central nervous system showed no significant findings. Mediastinal lymphoma was provisionally diagnosed.

\section{Histopathology}

Sections from different areas of the mediastinal mass and the adjacent infiltrated organs showed an inflammatory exudate consisting of macrophages, lymphocytes, plasma cells, and neutrophils. Areas of necrosis were also noted. Round and oval fungal organisms about 4-15 $\mu$ in diameter and with a surrounding clear halo (fig 2 ) were identified in routine haematoxylin-eosin stained sections. Inflammatory cells were sparse around groups or clusters of organisms. Using special stains, identification of the organisms as Cryptococcus neoformans became obvious. Mucicarmine stain (fig 3) clearly showed the gelatinous capsule of the organism. Absence of hyphae and the presence of budding forms were noted with Gomori's methenamine-silver nitrate stain. Sections from infiltrated areas of the lungs showed pneumonic consolidation with the presence of Cryptococcus neoformans. There was no evidence of lymphoma, tuberculosis, or other diseases in sections taken from the mediastinal mass or elsewhere.
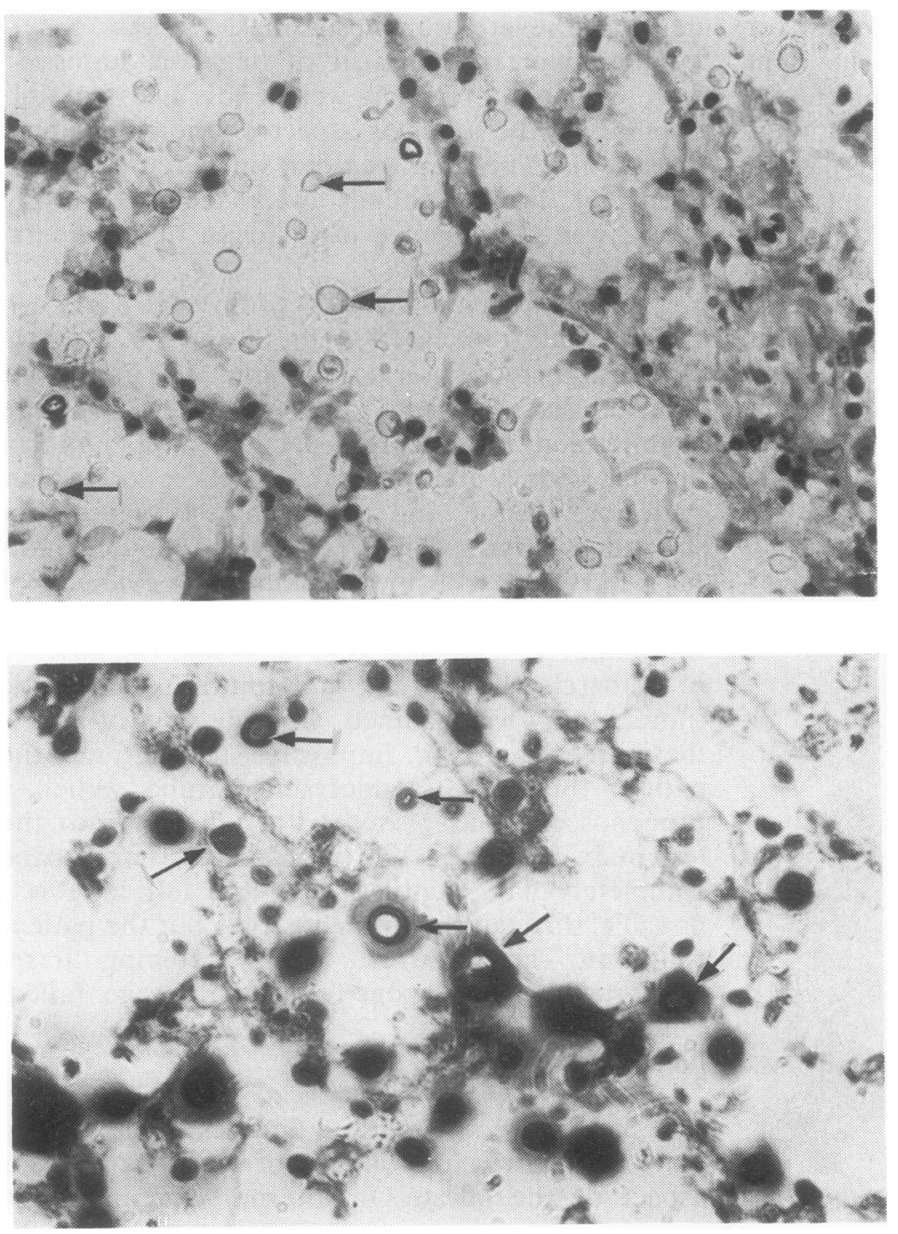

Fig 2 Section from mediastinal mass showing large numbers of cryptococci (arrowed) with surrounding clear halo and scanty inflammatory reaction (Haematoxylin and eosin $\times 345$ ).

Fig 3 The mucinous capsule (arrowed) of the Cryptococcus neoformans is shown (Mucicarmine stain $\times 345$ ). 


\section{Discussion}

Sporadic cases of cryptococcosis are known to occur throughout Africa (Bhagwandeen, 1969; Mpairwe and Patel, 1970; Gelfand, 1972; Siddorn, 1977). Pulmonary cryptococcosis masquerades as bronchial carcinoma or tuberculosis. The correct clinical diagnosis can sometimes be established by identification of the organisms in the sputum. Repeated examination of sputum on careful search, however, failed to show the organisms in the present case, a finding difficult to explain. Tuberculosis was suspected, and treatment was given without improvement. The operative and macroscopic necropsy findings were not suggestive of fungal disease, and the correct diagnosis was confirmed only by histopathological examination. Since mycosis was not suspected at necropsy, tissue was not collected for culture. Nevertheless, the morphological characteristics shown by routine haematoxylin-eosin and special stains fulfil the requirements outlined by Symmers (1953) and MacGillivary (1966) for identification of Cryptococcus in histology sections.
Cryptococcosis presenting as a mediastinal mass has not previously been reported.

We are grateful to Professor $\mathrm{L}$ Wasornu and $\mathrm{Mr}$ $\mathrm{R}$ Carruthers for invaluable advice and $\mathbf{M r} \mathbf{M} \mathbf{A}$ Ansary for the illustrations.

\section{References}

Bhagwandeen, S B (1969). Disseminated cryptococcosis. Medical Journal of Zambia, 2, 203-204.

Gelfand, M (1972). Cryptococcal meningitis. Central African Journal of Medicine, 18, 248-250.

MacGillivary, J B (1966). Two cases of cryptococcosis. Journal of Clinical Pathology, 19, 424-428.

Mpairwe, Y, and Patel, K M (1970). Cryptococcal meningitis in Mulago Hospital, Kampala. East African Medical Journal, 47, 445-447.

Siddorn, J A (1977). Unpublished data.

Symmers, W St C (1953). Torulosis. Lancet, 2, 10681076.

Requests for reprints to: P Sinha, FRCS, Department of Surgery, University Teaching Hospital, School of Medicine, University of Zambia, PO Box RW 110, Lusaka, Zambia.

\section{Calendar for Thoracic Society Meetings}

1979

1-3 February 1979

at Royal College of Physicians, London.

Members of American Thoracic Society are invited to attend this meeting.

6-7 June 1979

in Paris-joint meeting with French Thoracic Society.

1980

7-9 February 1980

at Royal College of Physicians, London (date to be confirmed).

3-4 July 1980

at Durham. Members of BTA and of the Belgian Thoracic Society are particularly invited to attend this meeting; details will be circularised.

For further details contact Dr S W Clarke, Department of Thoracic Medicine, Royal Free Hospital, Pond Street, London NW3 2QG. 